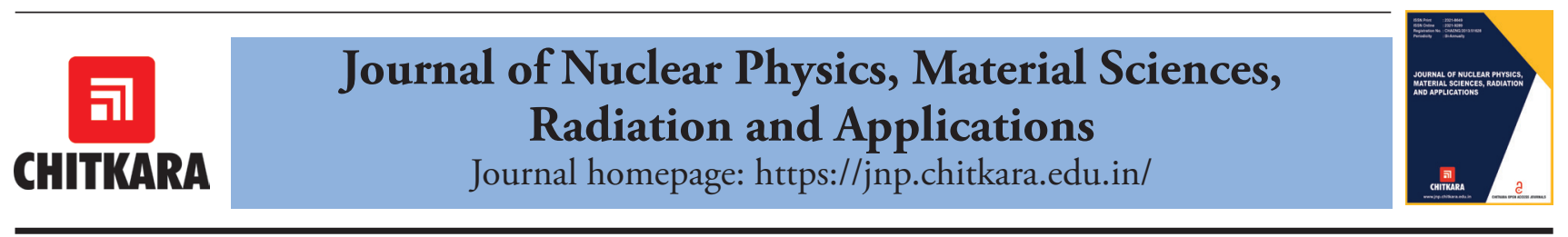

\title{
Behavior of Poly-A onto Kaolin
}

\begin{abstract}
María Guadalupe Torres-Duque ${ }^{1 *}$, Claudia Camargo-Raya ${ }^{2}$, Alicia Negrón-Mendoza ${ }^{2}$ and Sergio Ramos-Bernal ${ }^{2}$
${ }^{1}$ Faculty of Higher Education Iztacala, National Autonomous University of Mexico. Avenida de los Barrios Number 1, Colonia Los Reyes Iztacala, Tlalnepantla, State of Mexico

${ }_{2}^{2}$ Institute of Nuclear Sciences, National Autonomous University of Mexico. Circuito Exterior s/n, Ciudad Universitaria, Coyoacán, Mexico City
\end{abstract}

*Email: guadalupetduque@gmail.com

\section{ARTICLE INFORMATION}

Received: October 10, 2019

Accepted: January 29, 2020

Published online: February 28, 2020

Keywords:

Poly-A, Clays, Kaolin, Chemical evolution

DOI: $10.15415 /$ jnp.2020.72017

\begin{abstract}
A combination of geochemical variables is necessary to explain the origin of life on Earth. Thus, in this work the sorption of Poly-A on a clay mineral (kaolinite) was studied to get an insight about the sorption capacity at different times and $\mathrm{pH}$ values, as well as to confirm the capabilities of the clay to protect the sorbate from an external source of ionizing radiation. Poly-A presented a high percentage of sorption in the clay, especially in acidic environments, and this percentage sharply decrease in alkaline media. On the other hand, Poly-A's recovery was higher in the system with clay, confirming its protection role.
\end{abstract}

\section{Introduction}

Chemical evolution is a hypothetical process postulated to explain the formation of bio-organic molecules via the action of energy sources that could have caused chemical reactions in the primitive Earth and led to a period of synthesis and subsequent accumulation of organic material that preceded life's possible origin [1]. One hypothesis for the origin of life on Earth is a gradual transition from an environment of inorganic compounds yielding simple organic components (such as $\mathrm{CH}_{4}, \mathrm{NH}_{3}$, etc.) to an environment with complex biomolecules and polymers [2].

Experimental simulations of primitive Earth's possible conditions, in which all these events took place, help in studying the formation process of these molecules and could help us understand the first steps in the origin of life. One of the most important aspects is the relationship between chemical evolution and geologically relevant scenarios. In this regard, the contribution of solid surfaces-such as silicates, carbonates, sulfides and clays-may have been important [3].

Another consideration in chemical evolution is the source of energy for these processes. One main source of energy was ionizing radiation, which could have a critical role because of its high energy deposition and penetration power. This energy source interacts with chemical compounds to present changes that promoted an increase in organic material's complexity and diversity; it could also explain the decomposition patterns of some biomolecules [1].

In the chemical evolution process, the minerals present in the geological scenery, such as the clays, may have played a very important role in the formation of organic molecules due to their characteristics of absorbing, concentrating, protecting, and catalyzing the reactions of organic compounds [4].

Poly A is an important molecule in chemical evolution because it is an analog for nucleic acids in the steps from forming simple molecules to forming highly complex molecules, such as the informative-type RNA [5]. Gilbert proposed "the RNA world" an early period of Earth approximately 4 billion years ago in which RNA or some chemically similar molecule played a very important role in metabolic processes and in processing necessary information so other biologically important molecules and, later, the first simple organisms [6]. This molecule's catalytic and informative properties were key for processes that led to living beings. Coupled with this is the most attractive hypothesis, which suggests that adsorption to a specific surface of a mineral could be oriented by activating nucleotides and thus catalyzing a highly stereospecific reaction [7]. 
On the other hand, solid surfaces, such as clay minerals, may have an important role in prebiotic processes. The clays are constituted by aggregates of hydrated aluminum-silicates and have very variable compositions. With large surface and internal spaces or voids, their shape and properties depend on the type of clay in question [8].

Kaolin is a mineral found in the first ten meters of the continental crust. The kaolin is mainly composed of kaolinite, (sometimes halloysite), which is produced from mineral deposits with a significant amount of kaolin. It is a laminar silicate with two basic units that constitute its structure: $\mathrm{SiO}_{4}$ tetrahedra and $\mathrm{Al}_{2} \mathrm{O}_{3}$ octahedra. The silicon layer shares free vertices with the oxygen in the aluminum layer. The layers of silicon and aluminum fit together. The result is a double $\mathrm{Si} / \mathrm{Al}$ layer that is coupled with others to form the kaolin structure [9].

Minerals may have been key for prebiotic synthesis, leading researchers to include them in experiments carried out in heterogeneous systems exposed different energy sources. These studies focus on the behavior of biological molecules in the presence of some minerals to study the protective capacity they may provide under certain conditions [10]. In previous studies on the adsorption of nucleotides in clays such as montmorillonite, these mineral surfaces provided protection against radiation to prevent their degradation [11]. With RNA polynucleotides, sodium montmorillonite's protective capacity against external radiation sources has also been proven, increasing the chances of recovery of Poly-A compared to an absence of clay [12].

This work aims to test the capacity of adsorbing Poly A onto kaolin and to study the protector role of kaolin toward the irradiation of the Poly-A kaolin system at high doses of gamma irradiation.

\section{Materials and Method}

\subsection{Preparation of Solutions}

Using tridistilled water, Poly-A solutions (SIGMA Chemicals, USA) at a concentration of $5 \mathrm{mg} / 25 \mathrm{~mL}$ were prepared.

\subsection{Adsorption experiments}

For each solution, $0.10 \mathrm{~g}$ of kaolin obtained from the University of Missouri's Clay Minerals Repository in the USA was added to $3 \mathrm{~mL}$ aliquot solutions of Poly-A in a glass tube. The $\mathrm{pH}$ of the solutions was adjusted to 2, 4, 6, and 8 with $\mathrm{HCl}$ or $\mathrm{NaOH}$, and the tubes were stirred for 15 minutes then centrifuged at 26,000 rpm for 30 minutes. The supernatant was separated and analyzed to determine the adsorption percentage.

Desorption of the polynucleotide was performed by adding the clay to an aqueous solution of $\mathrm{NaOH}$ at
$\mathrm{pH} 8$ while the samples were continuously stirred and centrifuged. Adsorption and desorption supernatants were analyzed by spectroscopy with a UV-vis detector at $\lambda=260 \mathrm{~nm}$.

\subsection{Irradiation of samples}

At various times, irradiation was carried out in a ${ }^{60} \mathrm{Co}$ Gamma source (Gamma beam 651 PT) at ICN-UNAM at a dose rate of $158 \mathrm{~Gy} / \mathrm{min}$. The solutions were at $\mathrm{pH} 2$ in oxygen-free solutions and in the presence and absence of clay.

\subsection{Data Analysis}

The obtained percentages of the clay's adsorption and desorption at different $\mathrm{pH}$ ranges are presented in graphs showing the trends of polynucleotide behavior. After submitting the samples to the radiation, the dose/response relationship was observed, and the percentage amounts of unaffected Poly-A were compared to determine the kaolin's protective capacity.

\section{Results and discussion}

\subsection{Adsorption Experiments}

Changes in the $\mathrm{pH}$ of the solution affected the clay's adsorption capacity. Concerning the $\mathrm{pH}$ variations in the solution, 98\% of the Poly-A was adsorbed at $\mathrm{pH} 2$ and $40 \%$ at $\mathrm{pH}$ 8. This can be observed in the Figure 1 at fix agitation time. The tendency to decrease the adsorption percentage as the environment becomes more alkaline should be highlighted [13].

Figure 2 shows the adsorption in an acid medium. The kaolin presented a very high and significant adsorption capacity, with maximum adsorption levels greater than $95 \%$ in short agitation periods that did not exceed 15 minutes and remained constant.

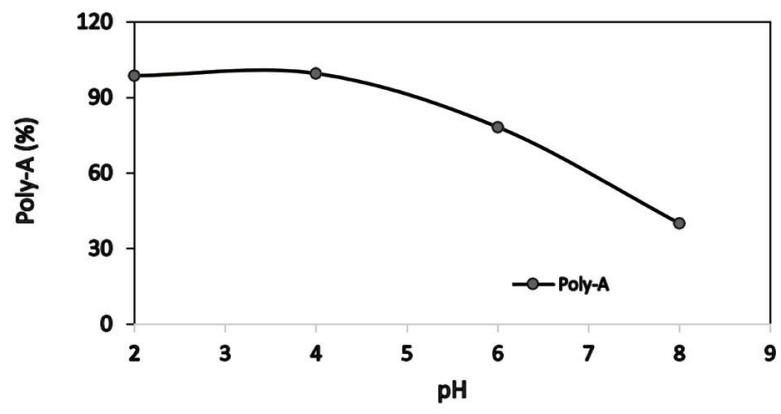

Figure 1: Adsorption of Poly-A at different $\mathrm{pH}$ and a fix agitation time. 


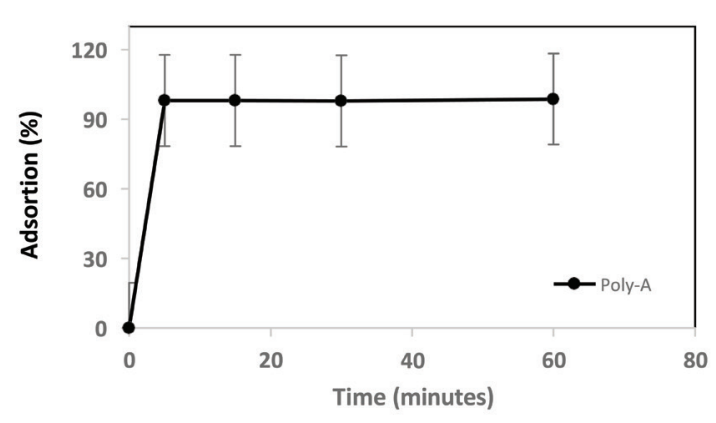

Figure 2: Adsorption of Poly-A on kaolin at pH 2.

Purines, pyrimidines, and nucleosides require adsorption conditions with an acidic $\mathrm{pH}$ around 2.5 [14]. Thus, to explain the adsorption capacity of the Poly-A system on kaolin, an acidic $\mathrm{pH}$ is necessary because the molecules are protonated at this $\mathrm{pH}$, and the adsorption occurs mainly in the octahedral surface hydroxyls via hydrogen bridges of the crystal or with the tetrahedral side through hydrogen bonds [13-15].

The mechanism of adsorption of organic molecules by means of clays is a complex phenomenon, one of these is that they act as an ion exchanger due to their excess surface charge. The adsorption depends on the conditions of the medium, such as the properties of the molecule, the concentration, the type of clay and the $\mathrm{pH}$, with respect to the latter it has been observed that in the case of bio-organic compounds the adsorption is greater in acidic conditions [16].

When treating the clays from previous samples for desorption, $50-65 \%$ of Poly-A was desorbed from the clay under alkaline conditions at $\mathrm{pH}$ 8. See Figure 3.

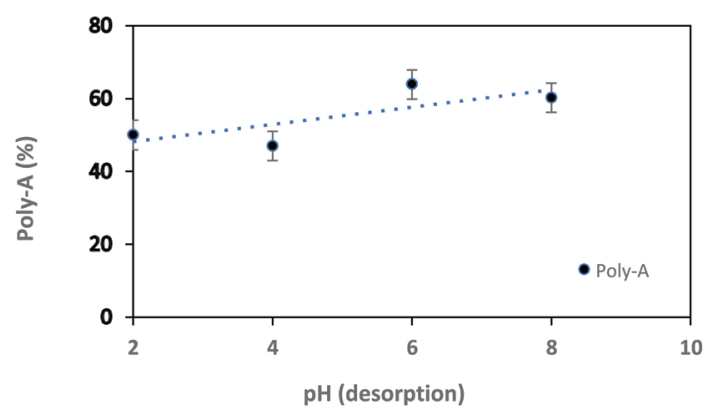

Figure 3: Poly-A desorption at different $\mathrm{pH}$ values.

\subsection{Irradiation Experiments}

The results indicate that Poly-A's degree of decomposition increases as the exposure time and gamma radiation dose increase, but the decomposition decreases in the presence of clay at the same irradiation dose as the samples without clay. The Poly-A was irradiated in different doses to observe the irradiation doses effect. Figure 4 shows that, at irradiation doses $16 \mathrm{kGy}, 71 \%$ of Poly-A was still observed, and almost $100 \%$ was present in the presence of clay, denoting that the kaolin can almost completely protect the molecule at that radiation dose. At $33 \mathrm{kGy}$, however, the solution without clay of Poly-A noticeably decomposes to $19 \%$; but with clay, a presence of $44 \%$ is recorded. This protective effect is still under study, and energy transfer processes may be the responsible for the observed behavior of the polymers in the kaolin at the surface, allowing the Poly-A to interact with the medium and providing only partial protection. Comparing these results with others obtained with montmorillonite where it has been observed that adsorption is carried out at the edges of the crustal structure with doses equal to those presented in this work [12].

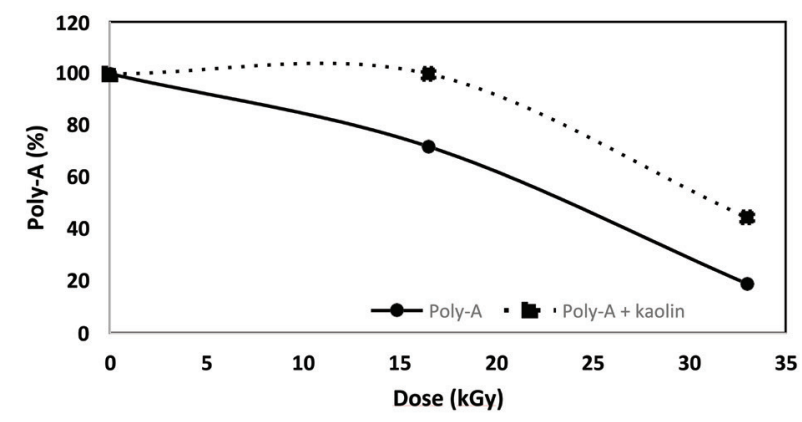

Figure 4: Radiolysis of Poly-A with and without kaolin (normalized values).

Kaolin has been shown to serve as a catalyst for the synthesis or polymerization of various types of organic molecules, including purines and pyrimidines [17].

\section{Conclusions}

Kaolin was shown to have a high adsorption capacity under acidic conditions for the Poly-A polymer and this capacity is affected by $\mathrm{pH}$. The clay has a partially protective capacity when Poly-A is exposed to a high energy source, as compared to Poly-A without the presence of the clay.

\section{Acknowledgments}

This work was performed at ICN-UNAM. The author(s) thank the PAPIIT Project IN226817 and PAPIIT IN110919, M. Sc B. Leal-Acevedo, Phys. F. Flores

\section{References}

[1] A. Negrón-Mendoza, S. Ramos-Bernal, M. ColinGarcía and A. Heredia, Radiation \& applications 1, 159 (2016). https://doi.org/10.21175/RadJ.2016.03.030 
[2] A. I. Oparin, The origin of life (MacMillan, New York, 1924), p. 109.

[3] S. Chang and N. Lahav, J. Mol. Evol. 8, 357 (1976). https://doi.org/10.1007/BF01739261

[4] J. D. Bernal, The Physical Basis of Life (Routledge y Kegan Paul, London, 1951), p. 364.

[5] W. Gilbert, Nature 319, 618 (1986). https://doi.org/10.1038/319618a0

[6] P. G. Higgs and N. Lehman, Nat. Rev. Genet. 16, 1 (2015). https://doi.org/10.1038/nrg3841

[7] S. Woodson and S. Mount, in The RNA world, edited by R. F. Gesteland, T. R. Cech and J. F. Atkins (Cold Spring Harbor Laboratory Press, USA, 1999), p. 709. https://doi.org/10.1101/cshperspect.a006742

[8] H. Hashizume, Clay Minerals in Nature (Intech Open, London, 2012), p. 197.

[9] H. H. Murray, M. S. Prasad and K. J. Reid, Appl. Clay Sci. 6, 87 (1991). https://doi.org/10.1016/0169-1317(91)90001-P

[10] L. López-Esquivel, A. Negrón-Mendoza, F. Mosqueira and S. Ramos-Bernal, Nucl. Instrum. Meth. A 619, 1 (2010).
[11] J. Ramírez-Carreón, S. Ramos-Bernal and A. NegrónMendoza, J Radioanal. Nucl. Chem. 318, 2435 (2018). https://doi.org/10.1007/s10967-018-6264-8

[12] N. Palomino-Aquino and A. Negrón-Mendoza, AIP Publishing 1671, 030007 (2015). https://doi.org/10.1063/1.4927196

[13] L. D. Perezgasga, F. G. Mosqueira, A. NegrónMendoza, L. De Pablo-Galán and A. Serrato-Díaz, Orig. Life Evol. Biospheres 35, 91 (2005). https://doi.org/10.1007/s11084-005-0199-0

[14] M. M. Mortland, Advances in Agronomy 22,75 (1970). https://doi.org/10.1016/S0065-2113(08)60266-7

[15] D. Tunega, G. Haberhauer, M. H. Gerzabek, H. Lischka, Langmuir 181, 139 (2002). https://doi.org/10.1021/la010914e

[16] Negrón-Mendoza and S. Ramos-Bernal, The role of clays in the origin of life. Origins: genesis, evolution and diversity of life (Kluwer Academic Publishers, USA, 2004), 181-194.

[17] E. T. Degens, G. R. Harvey and K. Mopper, Chemical Geology 9, 1 (1972).

https://doi.org/10.1016/0009-2541(72)90038-1 


\section{Journal of Nuclear Physics, Material Sciences, Radiation and Applications}

Chitkara University, Saraswati Kendra, SCO 160-161, Sector 9-C, Chandigarh, 160009, India

Volume 7, Issue 2

February 2020

ISSN 2321-8649

Copyright: [C 2020 María Guadalupe Torres-Duque et al.] This is an Open Access article published in Journal of Nuclear Physics, Material Sciences, Radiation and Applications (J. Nucl. Phy. Mat. Sci. Rad. A.) by Chitkara University Publications. It is published with a Creative Commons Attribution- CC-BY 4.0 International License. This license permits unrestricted use, distribution, and reproduction in any medium, provided the original author and source are credited. 